Thorax (1974), 29, 209.

\title{
Sudden death in patients awaiting coronary artery surgery
}

\author{
B. S. LEW IS a nd M.S. GOT S M N \\ Cardiac Unit, Wentworth Hospital and the University of Natal, Durban, South Africa
}

\begin{abstract}
Lewis, B. S. and Gotsman, M. S. (1974). Thorax, 29, 209-214. Sudden death in patients awaiting coronary artery surgery. Six patients with coronary artery disease are described who died suddenly while awaiting an operation to revascularize the myocardium. Each patient had recent onset of severe angina pectoris and this was caused by critical narrowing(s) of $90 \%$ or more of a major coronary artery(ies) supplying a large portion of the left ventricular myocardium. The syndrome can be diagnosed only by cardiac catheterization and high quality coronary arteriography.

Patients in whom the myocardial blood supply is critically compromised must be recognized. These patients are liable to sudden death and require urgent myocardial revascularization.
\end{abstract}

Selective coronary arteriography permits precise anatomical diagnosis of the morphology, distribution, and severity of coronary artery disease. Prognosis is closely related to the number of major vessels involved, to the severity of obstruction, and to the rate of progression of disease (Friesinger, Page, and Ross, 1970; Bemis, Gorlin, Kemp, and Herman, 1973; Bruschke, Proudfit, and Sones, 1973a, b). Narrowing of the left main or left anterior descending coronary artery carries a sinister prognosis, and patients with this lesion are liable to sudden death (Herrick, 1912; Cohen, Cohn, Herman, and Gorlin, 1972; Gotsman, Lewis, and Bakst, 1973).

This report describes the syndrome of sudden death in six patients who had critical obstruction of a major coronary artery and emphasizes the precarious state of these patients. Urgent myocardial revascularization must be undertaken when a major coronary artery is critically narrowed.

\section{CASE REPORTS}

The clinical, electrocardiographic, haemodynamic, and angiographic data are summarized in the Table.

PATIENT 1 A 54-year-old company director gave a four-year history of angina pectoris which had become severe during the month preceding admission; the pain was relieved by rest.

Physical examination was normal. Blood pressure was $128 / 80 \mathrm{mmHg}$. The electrocardiogram showed old diaphragmatic infarction with anterolateral ischaemia and the chest radiograph was normal.

Cardiac catheterization and cineangiocardiography were performed. The left ventricular pressure was $100 / 5-15 \mathrm{mmHg}$ and the end-diastolic pressure did not increase after angiography. Left ventriculography showed a small area of diaphragmatic asynergy (Gorlin, Klein, and Sullivan, 1967) and good overall ventricular function with an ejection fraction of $62 \%$. Coronary arteriography showed a small circumflex artery and complete occlusion of the right coronary artery. Myocardial perfusion depended on the ramus medianus (a large branch of the circumflex artery) and there was critical narrowing $(90 \%)$ at its origin (Table) (Fig. 1).

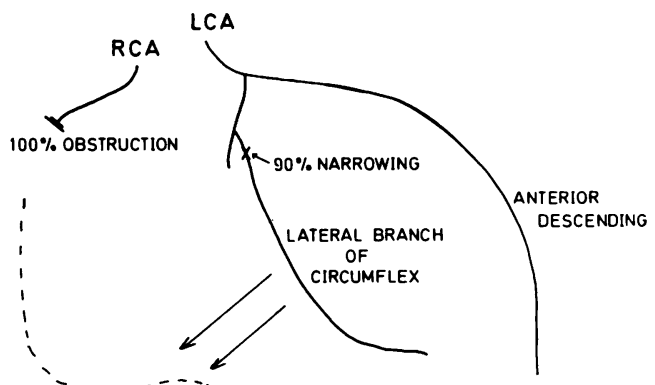

FIG. 1. Patient 1. Diagrammatic representation of the coronary arteriogram. There is complete obstruction of the proximal right coronary artery with late collateral filling of the distal vessel from a large branch of the circumflex artery. There is a $90 \%$ narrowing of this branch at its origin. 


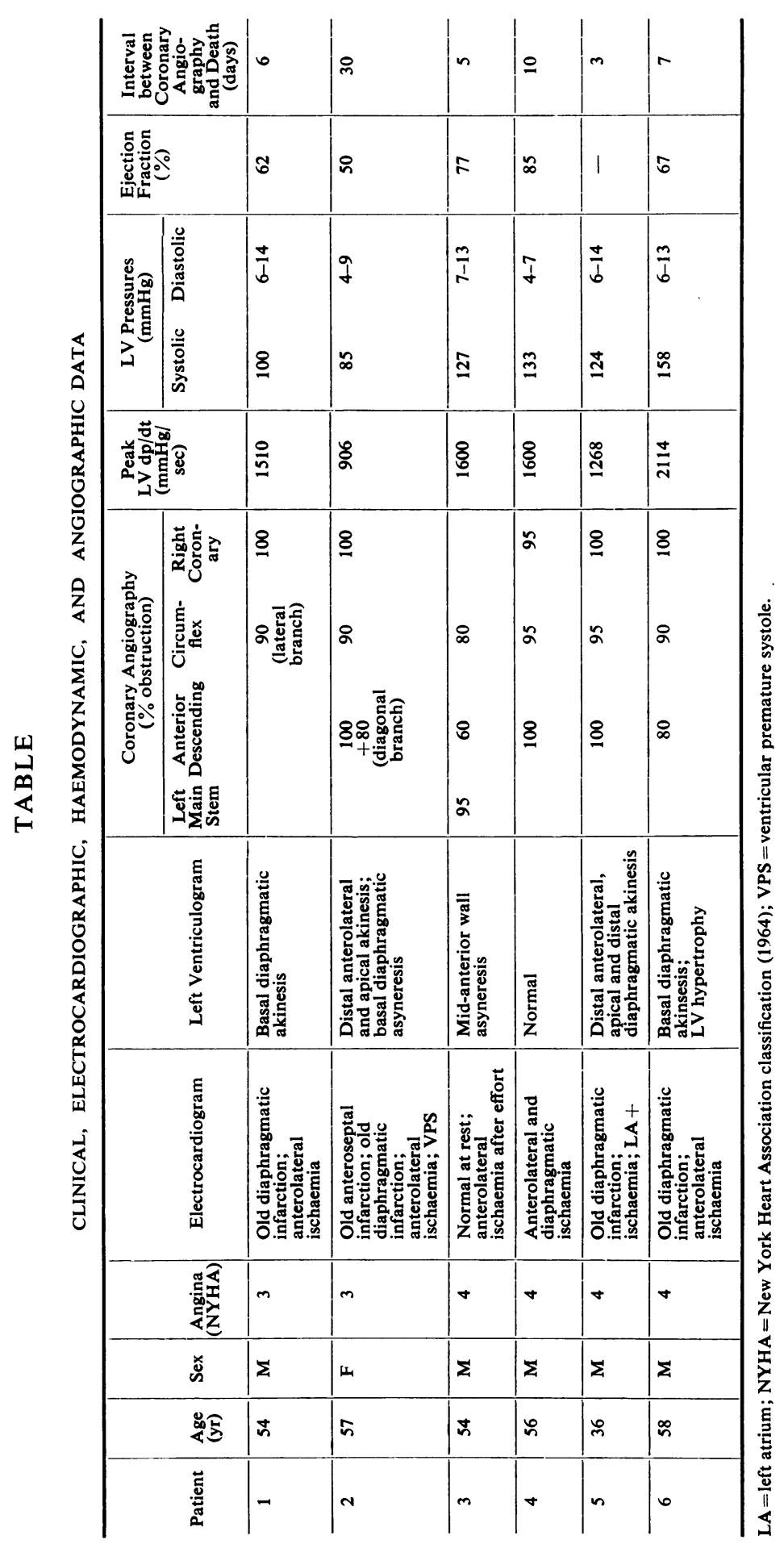


The patient was scheduled for the operation of double aortocoronary bypass grafting and was discharged for two weeks' bedrest while awaiting surgery: he died suddenly 12 hours after discharge from hospital.

PATIENT 2 A 57-year-old hospital matron gave a 10-day history of angina pectoris. Five years previously she had had one episode of myocardial infarction and since then had been asymptomatic.

On physical examination the blood pressure was $130 / 80 \mathrm{mmHg}$, the apical impulse was normal, and there was a fourth heart sound on auscultation. The electrocardiogram showed old anteroseptal and diaphragmatic infarction, anterolateral ischaemia, and several ventricular premature systoles. The chest radiograph was normal.

At cardiac catheterization the left ventricular pressure was $104 / 2-5 \mathrm{mmHg}$ and the end-diastolic pressure increased to $21 \mathrm{mmHg}$ after angiography. The left ventriculogram showed an enlarged left ventricle with impaired function: there was anterolateral and apical akinesis and additional asyneresis (diminished wall motion) of the basal diaphragmatic surface (Gorlin et al., 1967). The ejection fraction was $50 \%$. The right coronary artery and the anterior descending branch of the left were completely occluded. There was $90 \%$ segmental narrowing of the circumflex artery and $80 \%$ obstruction of the first diagonal branch of the anterior descending artery: these were large arteries which supplied collateral vessels to the distal anterior descending and the right coronary arteries (Fig. 2).

The severity of angina pectoris decreased during the period in hospital. The patient was discharged and was to return for elective myocardial revascularization and left ventricular aneurysmectomy: she died suddenly at home.

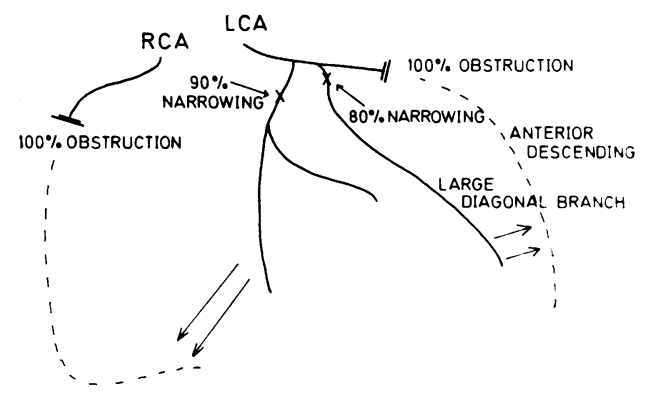

FIG. 2. Patient 2. Schematic representation of the coronary arteriogram. There is complete obstruction of the anterior descending and right coronary arteries with filling of the distal segments via collateral vessels. There is $80 \%$ narrowing of the main diagonal branch of the anterior descending artery and a $90 \%$ narrowing of the circumflex artery, the vessels from which collaterals arise.
PATIENT 3 A 54-year-old police captain had had angina pectoris for six months. His exercise tolerance decreased for a month before admission to hospital until angina was precipitated by climbing a few stairs.

On physical examination the blood pressure was $160 / 90 \mathrm{mmHg}$. A soft fourth heart sound was present at the apex. The resting electrocardiogram was normal but after effort there was pathological ST segment depression in the anterolateral leads. The chest radiograph was normal.

At cardiac catheterization the left ventricular pressure was $121 / 9-14 \mathrm{mmHg}$ with a peak $\mathrm{dp} / \mathrm{dt}$ of $1902 \mathrm{mmHg} / \mathrm{sec}$. The left ventriculogram was almost normal with a small segment of asyneresis involving the mid-anterior wall in late systole. The ejection fraction was $77 \%$. Selective coronary angiography showed a $95 \%$ obstruction of the main stem of the left coronary artery. There was also narrowing of the anterior descending branch $(60 \%)$ and of the circumflex artery $(80 \%)$. The distal vessels were normal. The right coronary artery was the seat of mild non-occlusive atheroma (Fig. 3).

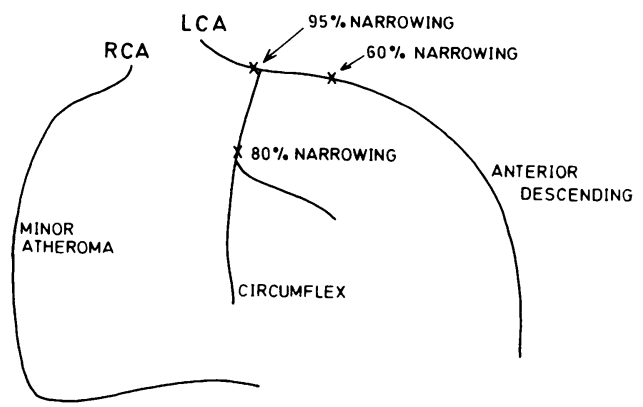

FIG. 3. Patient 3. Diagrammatic representation of the coronary arteriogram. There is a $95 \%$ narrowing of the main stem of the left coronary artery and additional narrowing of the anterior descending and circumflex branches.

The patient remained in hospital on strict bedrest. Coronary artery bypass surgery was scheduled. Five days after cardiac catheterization he developed severe chest pain, became hypotensive, and died within 10 minutes in ventricular fibrillation. Three doctors were in the ward precinct and nursing staff were in attendance to apply immediate external cardiac massage, ventilation, and intravenous therapy but the blood pressure remained unrecordable and resuscitation was abandoned after 30 minutes (Gotsman et al., 1973).

PATIENT 4 A 56-year-old business executive had had moderate angina pectoris for three years after an episode of acute myocardial infarction. For three months before admission to hospital the pain had 
become progressively more severe: he was grade 4 disabled, unable to climb steps, walk uphill or walk more than 30 yards on the flat. He also had nocturnal angina and episodes of pain at rest.

Physical examination revealed a normal blood pressure and a normal apical impulse. The electrocardiogram showed anterolateral and diaphragmatic ischaemia; the chest radiograph was normal.

Cardiac catheterization and cineangiography were performed. The left ventricular pressure was $133 / 4-7 \mathrm{mmHg}$ and peak dp/dt $1600 \mathrm{mmHg} / \mathrm{sec}$. The left ventriculogram was normal: ejection fraction was $80 \%$. Selective coronary angiography showed complete obstruction of the anterior descending artery, additional $95 \%$ narrowing of the circumflex artery, a large vessel, and also $95 \%$ narrowing of the right coronary artery. The distal vessels were well visualized and were normal (Fig. 4).

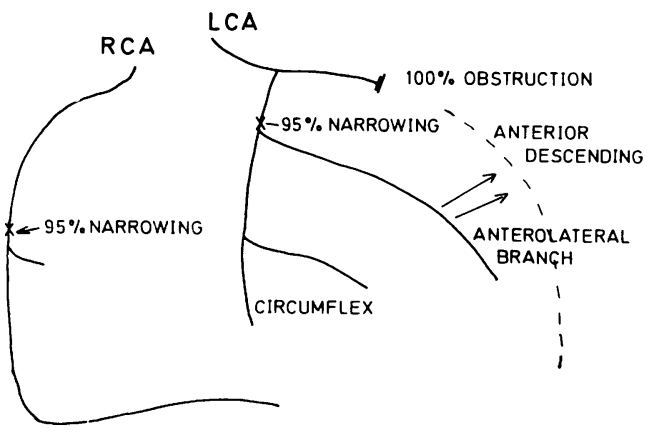

FIG. 4. Patient 4. Diagrammatic representation of the coronary arteriogram. The anterior descending artery is completely occluded and there is $95 \%$ narrowing of the circumflex artery before its main anterolateral branch. There is also $95 \%$ narrowing of the right coronary artery.

The operation of aortocoronary bypass grafting was scheduled. The patient requested to be allowed home for a weekend while awaiting surgery: he collapsed and died 48 hours after discharge from hospital.

PATIENT 5 A 36-year-old businessman complained of chest pain for one year and this had increased in severity for four to five months. Three days before admission to hospital he experienced repeated episodes of angina pectoris at rest and after minor exertion.

Physical examination was normal. The electrocardiogram showed old diaphragmatic infarction and anterolateral ischaemia; the chest radiograph was normal. Biochemical analysis showed a normal serum creatine phosphokinase (CPK), serum aspartate aminotransferase (SGOT), and lactic dehydrogenase (LDH) on repeated estimations. Serum cholesterol was increased $(300 \mathrm{mg} / 100 \mathrm{ml})$ and triglycerides were $\stackrel{\overrightarrow{\overrightarrow{\vec{S}}}}{\stackrel{\vec{\rho}}{\mathrm{E}}}$ high $(473 \mathrm{mg} / 100 \mathrm{ml})$.

Cardiac catheterization showed that the left $\frac{}{0}$ ventricular end-diastolic pressure was elevated $\overline{\bar{\rho}}$ (LV 119/4-14 mmHg). Peak LV dp/dt was nurmal $\mathbb{ه}$ $(1268 \mathrm{mmHg} / \mathrm{sec})$. The left ventriculogram showed akinesis of the apex and distal diaphragmatic surface $\infty$ of the left ventricle. Selective coronary arteriography $\overrightarrow{0}$ showed complete occlusion of two coronary arteries (right and anterior descending). There was a critical $\vec{\omega}$

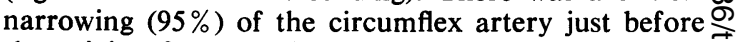
the origin of a large lateral branch (Fig. 5).

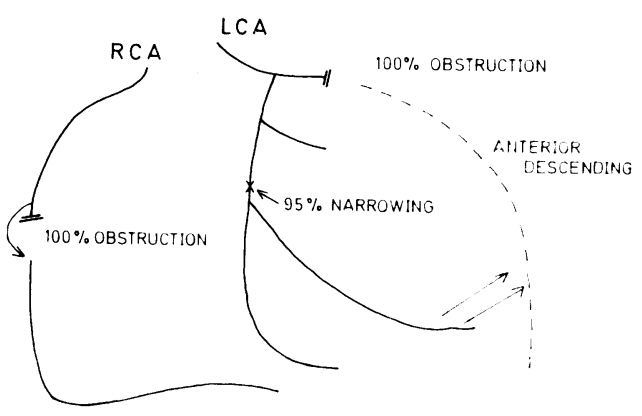

FIG. 5. Patient 5. Diagrammatic representation of $\overline{\bar{O}}$ the coronary arteriogram. There is complete obstruc-음 tion of the right and anterior descending coronary arteries, with $95 \%$ narrowing of the circumflex artery $\overrightarrow{\vec{B}}$ before its major ventricular branch.

An operation to revascularize the myocardium was planned. The patient developed chest pain and died suddenly three days after cardiac catheterization. $\frac{}{0}$ Serum enzyme analysis is performed as a routine in- $x$ vestigation for three successive days after coronary angiography: the enzymes were normal and death was not related to investigation.

PATIENT 6 A 58-year-old man had chest pain foro five months and this was followed by an episode of $\square$ acute myocardial infarction. He was hospitalized foro six weeks but the pain recurred throughout the period in hospital without a further change in the electro- $N$ cardiogram or serum enzymes.

On physical examination he was mildly hypertensive (blood pressure $140 / 100 \mathrm{mmHg}$ ), there was ac small ' $a$ ' wave in the jugular venous pressure, and an apical fourth heart sound. The electrocardiogramo showed left ventricular hypertrophy, old diaphrag- $\frac{-}{\Phi}$ matic infarction, and anterolateral ischaemia. Onc? the plain chest radiograph the left ventricle and aorta were enlarged.

Cardiac catheterization was performed. The mean $\vec{\Phi}$ aortic pressure was $120 \mathrm{mmHg}$ and the left ventricular $\frac{\stackrel{\rho}{\Phi}}{\mathrm{O}}$ pressure $158 / 6-13 \mathrm{mmHg}$ with an increased peak $\mathrm{LVdp} / \mathrm{dt}(2114 \mathrm{mmHg} / \mathrm{sec})$. The left ventriculogram showed a hypertrophied ventricle with good overall 
function and ejection fraction of $67 \%$ : there was akinesis of the basal half of the diaphragmatic surface. The coronary arteriogram showed triple vessel disease with complete right coronary obstruction, and $90 \%$ circumflex and $80 \%$ anterior descending artery narrowing. The distal vessels were normal (Fig. 6).

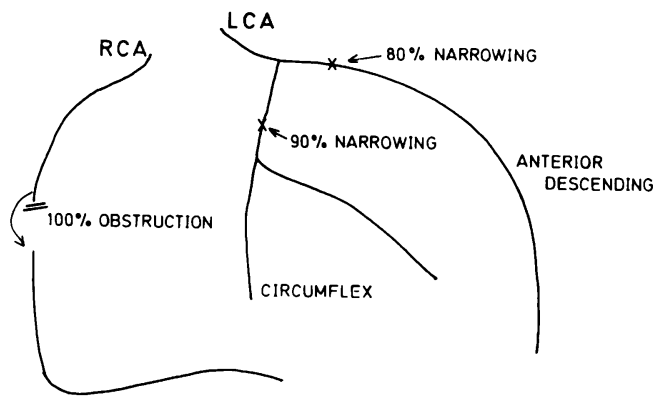

FIG. 6. Patient 6. Diagrammatic representation of the coronary arteriogram, showing complete obstruction of the right coronary artery, $80 \%$ narrowing of the anterior descending artery, and 90\% narrowing of the circumflex artery.

A triple aortocoronary bypass operation was scheduled. A week after catheterization the patient collapsed and died suddenly while resting in bed: attempts at resuscitation were unsuccessful.

\section{DISCUSSION}

Direct myocardial revascularization may relieve angina pectoris and prevent impending infarction (Proudfit et al., 1969; Effler, 1971; Effler, Favaloro, and Groves, 1971a; Effler, Favaloro, Groves, and Loop, 1971b; Rogers et al., 1972; Miller et al., 1973). Chronic angina, unresponsive to medical treatment, is an indication for operation, while the role of surgery in patients with unstable angina (preinfarction syndrome) is being studied. It has been suggested that operation should be undertaken in patients with severe proximal narrowing of a major coronary artery in the presence of moderate angina pectoris since the blood supply to a large area of myocardium is jeopardized and sudden death may occur (Favaloro et al., 1970).

We have described six patients with coronary artery disease who died suddenly. Each patient had critical narrowing of the major vessel supplying the left ventricular myocardium, either because of the underlying coronary anatomy or following complete obstruction of the other major coronary arteries. Overall left ventricular function was good in all the patients. In two patients left ventriculography showed no regional asynergy, in two asynergy was localized, while in the remaining two the extent of myocardial damage was greater. In each patient distal vessels suitable for myocardial revascularization were demonstrated. An operation date had been planned for each patient: three patients were discharged because of 'waiting list delay' and the other three were kept in hospital on strict bedrest after our initial experience. All three died suddenly in a fully equipped cardiac unit with highly trained nursing and medical staff in attendance. Immediate attempts at resuscitation failed because the entire left ventricular myocardium was ischaemic: pump failure or ventricular fibrillation, unresponsive to drugs or to electrical cardioversion, was inevitable.

The precise event causing myocardial infarction in a patient with coronary arterial disease is uncertain (Roberts, 1972). Patients with severe subtotal arterial narrowing are in a state of precarious balance and it is conceivable that a small change in myocardial oxygen supply or demand may be critical. Death occurred after cardiac catheterization and coronary angiography but each procedure was uneventful, the electrocardiogram was unchanged, and serial enzyme studies after the investigation were normal. Moreover, the time interval between investigation and sudden death suggested that the two events were not causally related.

Selective coronary angiography is an essential investigation in patients with severe angina pectoris. Patients with an acutely threatened myocardium must be recognized and operated on as soon as the diagnosis is made: the logistics of the problem seem overwhelming but delay may prove fatal.

\section{REFERENCES}

Bemis, C. E., Gorlin, R., Kemp, H. G., and Herman, M. V. (1973). Progression of coronary artery disease. A clinical arteriographic study. Circulation, 47, 455.

Bruschke, A. V. G., Proudfit, W. L., and Sones, F. M. Jr. (1973a). Clinical course of patients with normal and slightly or moderately abnormal coronary arteriograms. A follow-up study on 500 patients. Circulation, 47, 936.

,-- , and - (1973b). Progress study of 590 consecutive nonsurgical cases of coronary disease followed 5-9 years. I. Arteriographic correlations. Circulation, 47, 1147.

Cohen, M. V., Cohn, P. E., Herman, M. V., and Gorlin, R. (1972). Diagnosis and prognosis of main left coronary artery obstruction. Circulation, 45, Suppl. I, 57. 
Effler, D. B. (1971). Current era of revascularization surgery. Surgical Clinics of North America, 51, 1009.

, Favaloro, R. G., and Groves, L. K. (1971a). Myocardial revascularization. Journal of Cardiovascular Surgery Torino. 12, 1.

,,--- , and Loop, F. D. (1971b). The simple approach to direct coronary artery surgery. Journal of Thoracic and Cardiovascular Surgery, 62, 503.

Favaloro, R. G., Effler, D. B., Groves, L. K., Sheldon, W. C., Shirey, E. K., and Sones, F. M. Jr. (1970). Severe segmental obstruction of the left main coronary artery and its divisions. Journal of Thoracic and Cardiovascular Surgery, 60, 469.

Friesinger, G. C., Page, E. E., and Ross, R. S. (1970). Prognostic significance of coronary arteriography. Transactions of the Association of American Physicians, 83, 78.

Gorlin, R., Klein, M. D., and Sullivan, J. M. (1967). Prospective correlative study of ventricular aneurysm. American Journal of Medicine, 42, 512.

Gotsman, M. S., Lewis, B. S., and Bakst, A. (1973). Obstruction of the left main coronary arterythe artery of sudden death. South A frican Medical Journal, 47, 641 .
Herrick, J. B. (1912). Clinical features of sudden obstruction of the coronary arteries. Journal of the American Medical Association, 59, 2015.

Miller, D. C., Cannom, D. S., Fogarty, T. J., Schroeder, J. S., Daily, P. O., and Harrison, D. C. (1973). Saphenous vein coronary arterye bypass in patients with 'preinfarction angina' Circulation, 47, 234.

New York Heart Association (1964). Physical capacity. with heart disease. Diseases of the Heart and $\overrightarrow{.}$ Blood Vessels: Nomenclature and Criteria for Diagnosis, 6th ed., p. 110. Little Brown, New York.

Proudfit, W. L., Sones, F. M. Jr., Shirey, E. K. N Fergusson, D. J. G., and Sheldon, W. C. (1969). N Revascularization of the myocardium. Diseases of the Chest, 55, 315.

Roberts, W. C. (1972). Relationship between coronaryo thrombosis and myocardial infarction. Modern Concepts of Cardiovascular Disease, 41, 7.

Rogers, N. M. A., Bakst, A., Lewis, B. S., Moyes, D. G., and Gotsman, M. S. (1972). Early results⿳̄ of surgery for coronary artery disease. South African Medical Journal, 46, 1247.

Requests for reprints to: Dr. B. S. Lewis, Wentworth. Hospital, P.B. Jacobs, Natal. 